\title{
Article
}

\section{Redox-responsive micellar nanoparticles from glycosaminoglycans for CD44 targeted drug delivery}

Ana M. Carvalho, Raquel Teixeira, Ramon Novoa-Carballal, Ricardo A. Pires, Rui L. Reis, and Iva Pashkuleva Biomacromolecules, Just Accepted Manuscript • DOI: 10.1021/acs.biomac.8b00561 • Publication Date (Web): 14 May 2018

Downloaded from http://pubs.acs.org on May 15, 2018

\section{Just Accepted}

"Just Accepted" manuscripts have been peer-reviewed and accepted for publication. They are posted online prior to technical editing, formatting for publication and author proofing. The American Chemical Society provides "Just Accepted" as a service to the research community to expedite the dissemination of scientific material as soon as possible after acceptance. "Just Accepted" manuscripts appear in full in PDF format accompanied by an HTML abstract. "Just Accepted" manuscripts have been fully peer reviewed, but should not be considered the official version of record. They are citable by the Digital Object Identifier (DOI®). "Just Accepted" is an optional service offered to authors. Therefore, the "Just Accepted" Web site may not include all articles that will be published in the journal. After a manuscript is technically edited and formatted, it will be removed from the "Just Accepted" Web site and published as an ASAP article. Note that technical editing may introduce minor changes to the manuscript text and/or graphics which could affect content, and all legal disclaimers and ethical guidelines that apply to the journal pertain. ACS cannot be held responsible for errors or consequences arising from the use of information contained in these "Just Accepted" manuscripts. 
Redox-responsive micellar nanoparticles from

\title{
glycosaminoglycans for CD44 targeted drug delivery
}

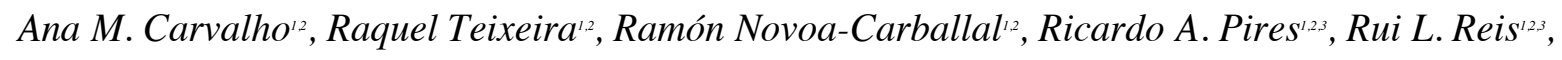 \\ Iva Pashkuleva ${ }^{12^{*}}$ \\ '3B's Research Group - Biomaterials, Biodegradable and Biomimetics, University of Minho, \\ Portugal \\ ${ }_{2}^{2}$ ICVS/3B's - PT Government Associate Laboratory, University of Minho, Portugal \\ ${ }^{3}$ The Discoveries Centre for Regenerative and Precision Medicine, Headquarters at University of \\ Minho, Avepark, 4805-017 Barco, Guimarães, Portugal
}

\section{KEYWORDS}

Hyaluronic acid; Chondroitin sulfate; CD44; self-assembly; micellar nanoparticles

\begin{abstract}
Cancer progression is associated with overexpression of various receptors at the cell surface. Among these, CD44 is known to recognize and bind specifically hyaluronan (HA) and interact with less affinity to other glycosaminoglycans (GAGs), such as chondroitin sulfate (CS). In this study, we describe a simple method to obtain micellar nanoparticles with GAGs shell (HA or CS) as potential drug delivery systems that target cancer cells overexpressing CD44. Alkane thiol was conjugated at
\end{abstract}


the reducing end of the respective GAG using highly efficient oxime chemistry. The alkane moiety confers amphiphilic behavior to the obtained conjugates and trigger their self-assembly into micellar nanoparticles, while the thiol group add redox-responsiveness to the system. The properties of the particles depend on the used GAG: HA amphiphiles form more dense, smaller assemblies that are redox sensitive. Both systems allow encapsulation of either hydrophobic or hydrophilic cargos with high efficiency. We demonstrate that the GAGs exposed on the surface of the nanoparticles are with preserved bioactivity and recognized by the cellular receptors: the particles were internalized via CD44 dependent pathways.

\section{INTRODUCTION}

Conventional cancer therapeutics aim selective cancer cell death and rely on exhaustive screenings of bioactive molecules that target different predefined hallmarks. ${ }^{1.3}$ Despite the undeniable success of this approach, numerous molecules that have shown great therapeutic potential in vitro are ineffective against tumors in vivo and cause several side effects. ${ }^{4}$ Usually, such failure is not associated with the pharmacodynamics (interaction of the drug with the target), but with the drug pharmacokinetics, biodistribution and bioaccumulation. ${ }^{3.5}$ Nanotechnology offers an opportunity to improve the efficacy of such molecules by enhancing their solubility and stability via encapsulation in biocompatible and non-toxic nanoparticles and also by modulation of the release process in terms of targeting, triggering, and kinetics using nanoparticles with precisely tailored composition..$^{57}$ In such strategies, the peculiar tumor microenvironment can be used as a target because it is abundant of various biomolecules with altered expression. It can be also used as a triggering stimulus because of the changed $\mathrm{pH}$ and redox status as compared to the microenvironment of healthy cells..$^{8 \cdot 12}$

Glycosaminoglycans (GAGs) are used as hallmark of different cancers: they are associated with cancer development, metastasis and invasiveness and exercise their role via interactions with specific 
membrane receptors that are also overexpressed in cancer cells. ${ }^{13.17}$ An example is CD44, the main hyaluronan (HA) receptor that also binds other GAGs although with less affinity ${ }_{.1822}^{10}$ In fact, HA-CD44 interactions are already used as targets in different anti-cancer strategies..$^{8.2326}$ The most common strategy involves the use of GAG (HA or chondroitin sulfate, CS) decorated carriers of different composition, shape and size that have loaded bioactive molecules. Such carriers improve the therapeutic efficacy of the cargo because of their ability to deliver it at the target, i.e. the cancer cells that are identified by the carriers via the CD44 overexpression. The incorporation of the GAG can be achieved by different approaches. Usually, the functionalization of the GAG is performed in side-on fashion via the $-\mathrm{COOH}$ groups of the uronic acid units..$^{10,22,24,27,28}$ Such approach can compromise the CD44 targeting as it alters dramatically the GAG flexibility, i.e. its ability to reorganize, adapt and bind multivalent to CD44; as well as its charge and polarity - the main driving forces in recognition processes..$^{29.31}$ Alternative endon functionalization at the GAG reducing end has been proposed recently to overcome this problem..$^{32}$. ${ }^{33}$ This approach involves modification of the HA by introducing alkyne function at the reducing end that can further participate in Huisgen cycloaddition with an azido functionalized counterpart. Block copolymers of HA generated by this approach self-assemble into polymersomes whose internalization by the cells is dependent on CD44 expression.

Herein, we propose an alternative, simple strategy for end-on functionalization of GAGs (HA and CS). We demonstrate that GAG amphiphiles can be obtained in one step from aminooxy alkane thiol and GAG at mild conditions via oxime ether formation. The generated amphiphiles self-assemble into micellar nanoparticles that can be loaded with either hydrophilic or hydrophobic cargos. The thiol groups confer redox-responsiveness of the assembled carriers. This feature of the nanoparticles is advantageous for cancer treatment because of the significantly higher concentration of glutathione (GSH) in the cytosol of tumor cells when compared to healthy ones., ${ }^{90}$

\section{MATERIALS AND METHODS}


Materials. Sodium hyaluronate (HA) from Lifecore (USA) and chondroitin sulfate (CS) from Kraeber (Germany) were used in this study. All other reagents (analytical grade) were purchased from Sigma-Aldrich and used as received.

Synthesis of glycosaminoglycan amphiphiles. 11-(Aminooxy)-1-undecanethiol (3 mol excess to GAG) was dissolved in $5 \mathrm{~mL}$ ethanol (for the synthesis of HA amphiphile) or $5 \mathrm{~mL}$ dimethyl sulfoxide (in the case of CS amphiphile), followed by the addition of $200 \mathrm{mg}$ of HA or CS dissolved in $5 \mathrm{~mL}$ acetate buffer ( $78 \mathrm{mM}$ acetic acid and $62.5 \mathrm{mM}$ sodium acetate, $\mathrm{pH} 4.5)$. The reaction mixture was magnetically stirred at $45^{\circ} \mathrm{C}$ during $24 \mathrm{~h}$. Unreacted reagents were removed by dialysis against ethanol (cut-off $14 \mathrm{kDa}$ ). The product was obtained by ethanol evaporation followed by freeze-drying.

Nuclear Magnetic Resonance spectroscopy (NMR). 'H NMR spectra were recorded on a Bruker Avance $400 \mathrm{MHz}$ spectrometer at $25^{\circ} \mathrm{C}$ or $60{ }^{\circ} \mathrm{C}$ in appropriated solvent $\left(\mathrm{D}_{2} \mathrm{O}\right.$ or DMSO- $\left.\mathrm{d}_{6}\right)$. Chemical shifts are reported in ppm ( $\delta$ units) downfield from 3-(trimethylsilyl)-propionic acid- $\mathrm{d}_{4}$.

Gel Permeation Chromatography (GPC). GPC measurements were performed with a Malvern Viscotek TDA 305 with refractometer (RI-Detector 8110, Bischoff), right angle light scattering (LS) and viscometer detectors on a set of four columns: pre-column Suprema $5 \mu \mathrm{m} 8 \times 50 \mathrm{~S} / \mathrm{N} 3111265$, Suprema $30 \AA 5 \mu \mathrm{m} \mathrm{8x300} \mathrm{S/N} \mathrm{3112751,} \mathrm{Suprema} 1000 \AA 5 \mu \mathrm{m} 8 \times 300$ S/N 3112851 PL and AquagelOH MIXED $8 \mu \mathrm{m}$ 7.5x300 S/N 8M-AOHMIX-46-51..$^{34}$ The system was kept at $30{ }^{\circ} \mathrm{C}$. We used $0.1 \mathrm{M}$ $\mathrm{NaN}_{3}, 0.01 \mathrm{M} \mathrm{NaH}_{2} \mathrm{PO}_{4}(\mathrm{pH}=6.6)$ as an eluent at a flow rate of $1 \mathrm{~mL} / \mathrm{min}$. The molecular weight of the GAGs was determined with a conventional (RI vs elution time) and multidetector calibration (RI+LS+Viscosity) using the software Omnisec 4.6.1 (ViskoteK). The conventional calibration was performed with commercial polysaccharide set from Varian that contains 10 pullulans with narrow polydispersity and molecular mass at the peak maximum (MP) ranging from $180 \mathrm{Da}$ to $708 \mathrm{kDa}$. The multidetector calibration was performed using the pullulan of this set with number-average molecular weight (Mn) $48.8 \mathrm{kDa}$ and PDI 1.09 . 
Determination of critical micelle concentration (CMC). CMC of GAG amphiphiles was determined according to previously described method using the hypsochromic shift of emission spectra of Nile Red probe in the presence of the amphiphiles at different concentrations ${ }^{35}$ Briefly, solutions of GAG amphiphiles (ultrapure water) with different concentrations $(0.02-15 \mathrm{mg} / \mathrm{mL})$ were incubated with Nile Red $\left(2 \mu \mathrm{M}\right.$ in ethanol) at $45^{\circ} \mathrm{C}$ for 45 minutes. Fluorescence spectra of these solutions were recorded on fluorescence spectrometer FP-8500 (Jasco) after cooling $\left(25^{\circ} \mathrm{C}\right)$. Nile Red was excited at $565 \mathrm{~nm}$ and emission spectra were recorded between 590 and $750 \mathrm{~nm}$. The slit width was set at $5 \mathrm{~nm}$ for both excitation and emission. The peak intensity at $615 \mathrm{~nm}$ was recorded as a function of the logarithm of GAG amphiphile concentration. CMC was determined at the interception of the two logarithmic equations.

Size and zeta-potential of micellar nanoparticles. GAG amphiphiles were dissolved in ultrapure water at concentration above the $\mathrm{CMC}$ and vortexed. Hydrodynamic diameter and zeta potential were determined by dynamic light scattering (DLS) and electrophoretic light scattering (ELS) using NANO ZS (Malvern Instruments Ltd) equipped with a $4 \mathrm{~mW}$ He-Ne laser operating at a wavelength of 633 nm. The scatter light was detected at a back-scatter angle $\left(173^{\circ}\right)$, which provides the sensitivity required for measuring particles at low concentrations. Measurements were carried using a polystyrene cell at $25^{\circ} \mathrm{C}$. The CONTIN algorithm (intensity weighted) was applied to obtain the size distribution. The average hydrodynamic diameter (Dh) and polydispersity index (PDI) were determined by fitting the correlation function with the cumulant method. Particles stability was evaluated by monitoring the polydispersity index and $\zeta$-potential over the time.

Diameter was also measured by Atomic Force Microscopy (AFM). A droplet of sample aqueous solution was deposited on an aminated freshly cleaved mica surface.$^{36}$ The surface was dried under a nitrogen flux. Measurements were performed with NanoWizard 3 (JPK, Germany) in quantitative imaging mode using silicon cantilevers (ACSTA, AppNano, USA) with a typical resonance frequency 
of $150 \mathrm{kHz}$ and a spring constant of about $7.8 \mathrm{~N} \mathrm{~m}^{-1}$. Size distribution was obtained after analyzing at least 150 particles using the software Gwyddion.

Redox-responsiveness of the micellar nanoparticles. The redox-responsiveness was evaluated by fluorescence quenching of Nile Red in the presence of 1,4-dithiothreitol (DTT). Nanoparticles containing Nile Red were prepared as described above. Solutions of GAG amphiphiles at a concentration above CMC $(2.4 \mathrm{mg} / \mathrm{mL} \mathrm{HA}$ and $6 \mathrm{mg} / \mathrm{mL} \mathrm{CS})$ containing $5 \mu \mathrm{M}$ of Nile Red were incubated with DTT of different concentrations $(50-200 \mathrm{mM})$ at $37{ }^{\circ} \mathrm{C}$. The fluorescence was measured by fluorescence spectrometer FP-8500 (Jasco) at $25{ }^{\circ} \mathrm{C}$. The excitation at $565 \mathrm{~nm}$ was performed and emission spectra were recorded in the range $590-750 \mathrm{~nm}$. The slit width was set at 5 $\mathrm{nm}$ for both excitation and emission. Stern Volmer constant was determined $\left(K_{s v}\right)$ by fitting the equation (1) to the experimental data

$$
K_{a p p}=K_{S V}+K_{D} K_{S}[Q]
$$

where $K_{a p p}$ is the apparent quenching constant, [Q] is DTT concentration, $\mathrm{K}_{\mathrm{D}}$ and $\mathrm{K}_{\mathrm{s}}$ are the dynamic and static components of the fluorescence quenching.

Stability of the micellar nanoparticles in the presence of DTT was evaluated by DLS. GAG amphiphiles $(2.4 \mathrm{mg} / \mathrm{mL} \mathrm{HA}$ and $6 \mathrm{mg} / \mathrm{mL} \mathrm{CS})$ were mixed with DTT of different concentrations $(0$ - $200 \mathrm{mM}$ ) and analyzed by DLS using NANO ZS (Malvern Instruments Ltd) with fixed position and attenuator.

Encapsulation efficiency. Fluorescein isothiocyanate-dextran (FITC:Glucose 1:250, MW 4000 g/mol) and Nile Red were used as model hydrophilic and hydrophobic drug, respectively. FITC:Glucose or Nile Red (2 mol\%) was added to micellar nanoparticles and the samples were incubated at $45{ }^{\circ} \mathrm{C}$ during 45 minutes. The free (non-encapsulated) compounds were separated by centrifugation using ultracentrifuge units with cut-off of $30 \mathrm{kDa}$ in a Gyrospin centrifuge scanspeed Mini (Labogene) at $6000 \mathrm{G}$ for 20 minutes. The supernatants were quantified using fluorescence 
spectrometer FP-8500 (Jasco). Encapsulation efficiency (\% EE) was determined according to the equation (2)

$$
\% E E=\frac{\left[C_{\text {total }}\right]-\left[C_{\text {free }}\right]}{\left[C_{\text {total }}\right]} \times 100
$$

where $\mathrm{C}_{\text {vatl }}$ is the concentration of probe in solution and $\mathrm{C}_{\mathrm{frec}}$ the concentration of the non-encapsulated probe.

Internalization assay. We used two human breast cancer cell lines, namely MDA-MB-468 and SKBR-3, for these experiments. The cells were seeded on tissue culture polystyrene at $37{ }^{\circ} \mathrm{C}$ in Dulbecco Modified Eagle Medium (DMEM) high glucose with phenol red (Sigma-Aldrich, St. Louis, UK) supplemented with sodium bicarbonate, $10 \%$ fetal bovine serum (FBS) and 1\% antibiotic/antimycotic. Upon confluence, the cells were detached with TrypLE ${ }^{\mathrm{TM}}$ and cultured in fresh medium at cell density of $20^{s} \mathrm{cell} / \mathrm{cm}^{2}$ overnight. Blocking of CD44 was performed by treatment of the cells with $150 \mathrm{ng} / \mathrm{mL}$ of anti-CD44 antibody [KM201] in DMEM without phenol red and FBS for $1 \mathrm{~h}$ at $37^{\circ} \mathrm{C}$. Non-specific blocking was carried out at the same conditions using $150 \mathrm{ng} / \mathrm{mL}$ mouse IgG1, kappa monoclonal antibody (MOPC-21).

The respective amphiphile (HA or CS) was dissolved in ultrapure water at concentration $3 \mathrm{mg} / \mathrm{mL}$. The assembled micellar nanoparticles were incubated with Nile Red $(1 \mu \mathrm{M})$ at $45{ }^{\circ} \mathrm{C}$ with orbital shaking for $2 \mathrm{~h}$. After cooling down to room temperature, the nanoparticles were filtered $(0.22 \mu \mathrm{m})$ and added to DMEM at a volume ratio 1:10. Treated and untreated cells were supplemented with this medium and incubated for $4 \mathrm{~h}$ at $37^{\circ} \mathrm{C}$ in humidified $5 \% \mathrm{CO}_{2}$ atmosphere. After this period, the cells were washed with phosphate buffer saline (PBS) and fixed with $10 \%$ formalin at $4{ }^{\circ} \mathrm{C}$. Cell membrane was stained with wheat germ agglutinin Alexa Fluor ${ }^{\circledR} 488$ conjugate in PBS $(1 \mu \mathrm{g} / \mathrm{mL})$ for 15 min at room temperature. After washing, cells were permeated with $0.2 \%$ Triton $\mathrm{X}$ and nuclei were stained with DAPI (1 $\mu \mathrm{g} / \mathrm{mL})$ for $30 \mathrm{~min}$. Coverslips with the fixed cells were mounted with vectashield 
mounting medium. Fluorescent images were acquired in Inverted confocal microscope TCS-SP8 (Leica, Germany).

\section{RESULTS AND DISCUSSION}

Synthesis and characterization of amphiphilic GAGs. The feasibility and versatility of oxime coupling for the synthesis of different glycoconjugates has been already demonstrated ${ }^{31,3739}$ Previous studies showed that this coupling preserves GAG bioactivity and specificity towards proteins, such as growth factors. .0, $^{39}$ Herein, we obtained amphiphilic GAG derivatives from 11-(Aminooxy)-1undecanethiol (Supplementary Information, SI, Scheme S1) and the respective GAG (HA or CS) by oxime coupling at the GAG reducing end (Fig. 1). Of note, the aminooxy alkanethiol was generated in situ from its protected derivative (SI, Scheme S1, 3) to avoid side reactions due to the observed high reactivity of the aminooxy group. The successful synthesis was confirmed by NMR (Fig. 1B; SI Fig. S4) and GPC (Table 1; SI Fig. S5).

The degree of substitution was determined from 'H NMR by the integral's ratio of the peaks corresponding to the aliphatic protons $\left(\delta_{\text {сн2 }} 1.25 \mathrm{ppm}\right)$ and the ones assigned to the protons of the glucuronic acid unit $\left(\delta_{\mathrm{н}} 3.4 \mathrm{ppm}\right)$. We obtained $99 \%$ substitution for HA amphiphile and $85 \%$ for CS derivative.

We expected that the modification of GAG will result in very small shift towards higher molecular weight (the molecular weight of the amphiphiles differ from the respective GAG with $c a .219 \mathrm{~g} / \mathrm{mol}$ ). Indeed, the amphiphilic derivatives have higher weight-average molecular weight (Mw) and Mn than the GAGs (Table 1; SI Fig. S5). Significantly different Mn was determined for the CS derivative. This difference is caused by the presence of assemblies that results in the appearance of an additional peak at lower retention times (SI Fig. S5E, orange). The assemblies' fraction is very small as it is detectable only by the light scattering (RALS) detector but not by the refractive index (RI) one. This secondary peak is not present in the GPC elugram of the HA amphiphile either below or above its CMC. Two 
A
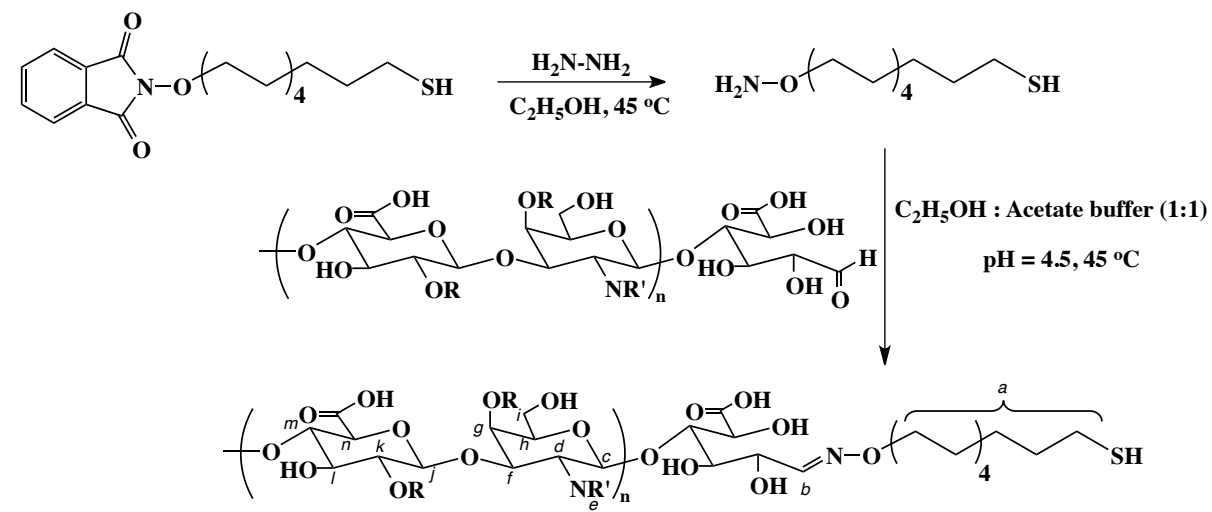

$\mathrm{R}=\mathrm{H}, \mathrm{SO}_{3} \mathrm{H} ; \mathrm{R}^{\prime}=\mathrm{H}, \mathrm{Ac}\left(\mathrm{CH}_{3} \mathrm{CO}\right)$
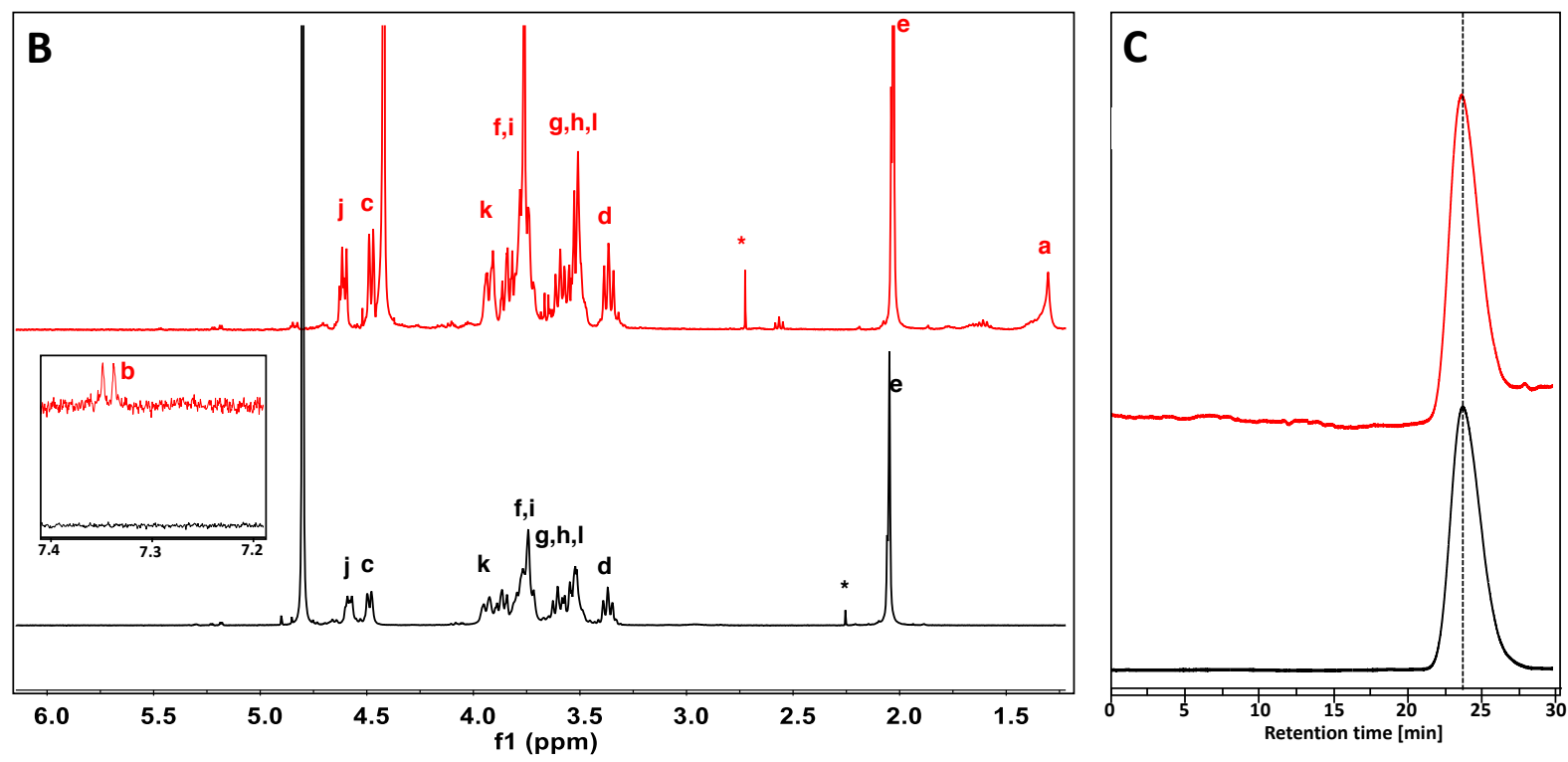

Figure 1. (A) Reaction scheme used for the synthesis of GAG amphiphiles and characterization of hyaluronan (black) and its derivative (red) by (B) 'H NMR and (C) GPC chromatogram (refractive index signal). Signals marked with (*) correspond to impurities from the solvent. NMR and GPC data for chondroitin sulfate and its derivative are provided in SI. 
Table 1. Number-average molecular weight $(\mathrm{Mn})$ and weight-average molecular weight $(\mathrm{Mw})$ of GAGs and their amphiphiles determined by gel permeation chromatography (GPC).

\begin{tabular}{lllll}
\hline & Mn [kDa] & Mw [kDa] & PDI & Retention time [min] \\
\hline HA & 3.30 & 4.3 & 1.37 & 23.7 \\
HA amphiphile & 4.3 & 5.2 & 1.20 & 23.6 \\
CS & 12.3 & 19.2 & 1.56 & 22.2 \\
CS amphiphile & $18.5^{*}$ & $23.1^{*}$ & 1.25 & $22.2(+18.8 \mathrm{LS})$ \\
\hline
\end{tabular}

*Increased values for Mn and Mw determined due aggregation (see also Suppl Fig. S5)

Assembly of GAG amphiphiles. Amphiphile molecules have tendency to self-organize in solution because of the thermodynamic incompatibility between their building blocks. As a result, different nanostructures (e.g. micelles, vesicles, nanofibers, lamellae) can be generated. The size and the architecture of the assemblies depend on the molecular weight of the hydrophilic and the aliphatic portion, the ratio between them and the environment (e.g. solvent, temperature). The assembly of the obtained GAG amphiphiles was studied in water either at room temperature or at $37^{\circ} \mathrm{C}$. Atomic force microscopy (AFM) showed that these molecules self-organize in nanostructures with round shape (Fig. 2C). The size of the assemblies is very important for drug intracellular delivery: sizes bellow $200 \mathrm{~nm}$ are associated with low reticuloendothelial uptake, minimal renal excretion, and effective enhanced permeation and retention (EPR) effect that favors passive tumor targeting. ${ }^{\text {. }}$ Previous studies with sideon modified GAGs report different diameter ranging from $37 \mathrm{~nm}$ to $500 \mathrm{~nm} .^{28,4044}$ DLS analysis of the assemblies of GAG amphiphiles obtained by us showed that they have Dh below $200 \mathrm{~nm}$ and low PDI (Table 2). The size depends on the GAG moiety in the amphiphile: HA amphiphiles form smaller assemblies whose size decreases with the temperature increase. The assemblies generated from the CS amphiphile are bigger and their size does not depend on the temperature. 
Table 2. Characterization of the formed assemblies in terms of size (DLS), charge (ELS) and critical micelle concertation (CMC, fluorescence spectroscopy of Nile Red).

\begin{tabular}{llllll}
\hline & $\mathbf{T}\left[{ }^{\circ} \mathbf{C}\right]$ & $\mathbf{D}_{\text {н }}[\mathbf{n m}]^{*}$ & PDI* & $\begin{array}{l}\zeta \text {-potential } \\
{[\mathbf{m V}]}\end{array}$ & $\mathbf{C M C}$ \\
& & & & & {$[\mu \mathrm{g} / \mathbf{m L}]$} \\
\hline HA amphiphile & 25 & $146 \pm 7.4$ & $0.20 \pm 0.01$ & $-31.9 \pm 1.7$ & $310.17 \pm 38.22$ \\
& 37 & $99 \pm 57.2$ & $0.20 \pm 0.01$ & $-34.9 \pm 3.1$ & $349.50 \pm 107.22$ \\
& 42 & & & & $287.67 \pm 5.86$ \\
CS amphiphile & 25 & $193 \pm 8.10$ & $0.16 \pm 0.02$ & $-25.1 \pm 2.8$ & $103.48 \pm 17.24$ \\
& 37 & $195 \pm 10.99$ & $0.14 \pm 0.02$ & $-31.6 \pm 0.1$ & $80.50 \pm 16.33$ \\
& 42 & & & & $96.47 \pm 2.95$
\end{tabular}

*Data reported as averaged values from at least three different measurements/batches; Used abbreviations: $\mathrm{T}$ temperature; $\mathrm{D}_{\mathrm{H}}$ hydrodynamic diameter; PDI polydispersity index

There are two reasons behind the obtained results: (i) different solvation effect and Coulomb forces and (ii) different molecular weights of the used GAGs (Table 1). HA is a weak polyelectrolyte, whereas CS has a higher charge density/repulsion caused by the sulfate groups and longer chains..$^{17,45}$ As a consequence, HA derivatives form smaller and more compact assemblies than the CS ones. The temperature-dependent size of the HA assemblies can be explained by the ability of this GAG to form H-bonding with water molecules; higher temperature results in decreased hydration enabling the formation of intermolecular H-bonding that lead to a decrease of the size of HA assemblies.

Previous reports about end-on functionalized HA describe formation of polymersomes with a hydrodynamic diameter around $440 \mathrm{~nm} \cdot .^{40}$ The structure of the amphiphiles obtained by us is different (large GAG portion and short aliphatic moiety) and the size that we have obtained is much smaller than the reported one. These differences are consistent with micellar organization in which the GAG part is exposed on the surface conferring colloidal stability and assuring targeted delivery, while the hydrophobic portion is hidden in the assembly interior (Fig. 2B). Indeed, the negative $\zeta$-potential confirm the presence of the GAG on the surface of the assemblies (Table 2). The formation of such 
structures has been observed in self-assembled HA with poly(DL-lactide-co-glycolide) (PLGA) copolymers. ${ }^{46}$ The end-on functionalization of HA $(7.5 \mathrm{kDa})$ with PLGA $(5 \mathrm{kDa})$ through disulfide linkage as resulted in self-assembled micellar nanoparticles with hydrodynamic diameter below 200 nm.
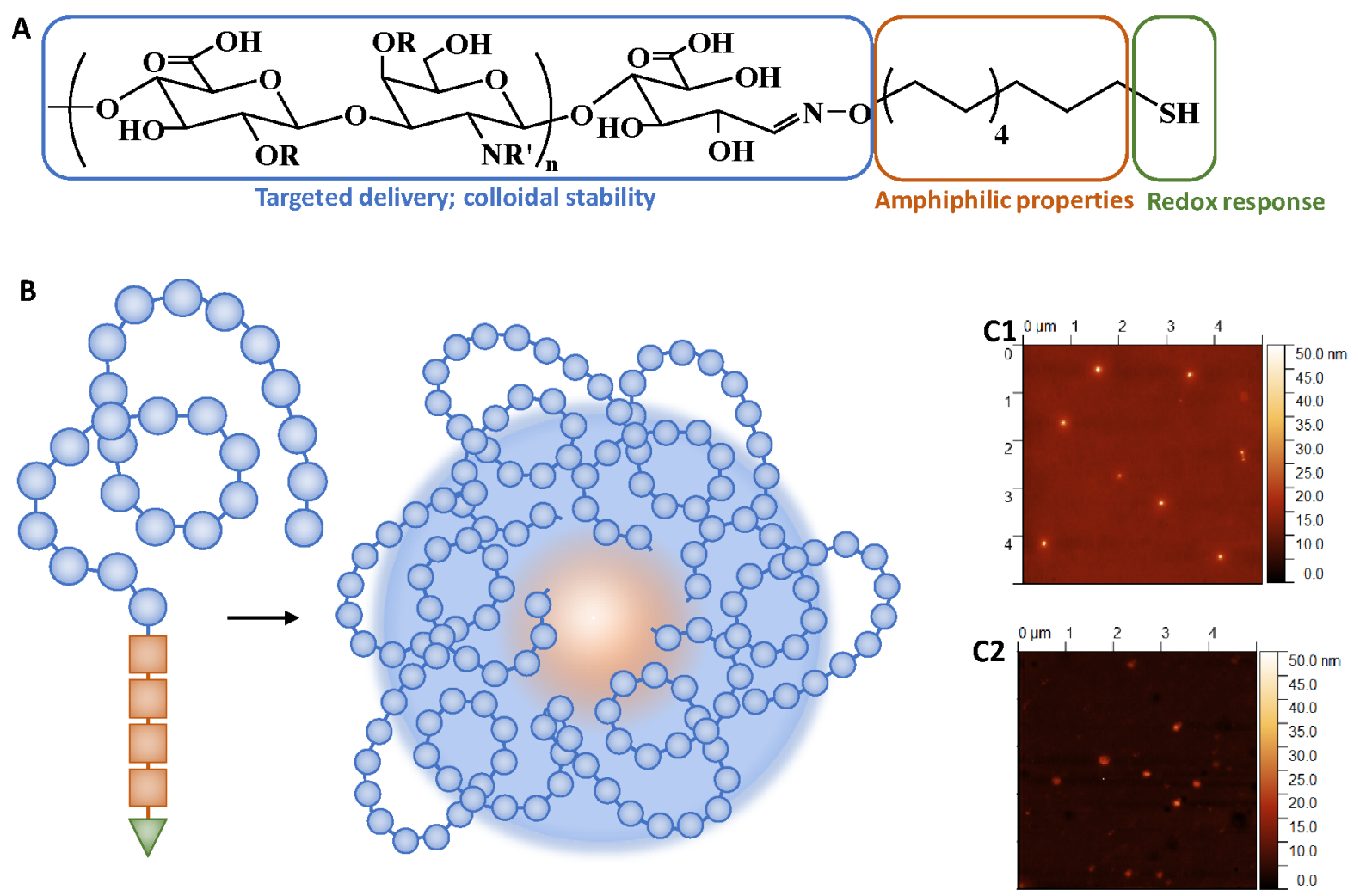

Figure 2. (A) Schematic presentation of the building blocks of GAG amphiphiles and their key role(s) in (B) the assembled micellar nanoparticles. (C) AFM images of the nanoparticles obtained from the (C1) hyaluronate and (C2) chondroitin sulfate amphiphiles.

Critical micelle concentration (CMC) is a key parameter related with the assembly and stability of the micellar nanoparticles. We used fluorescence spectroscopy of Nile Red to determine CMC (Table 2). ${ }^{35.47}$ Low fluorescence intensity of Nile Red was observed at lower concentration of GAG amphiphiles consistent with free molecules in solution (SI Fig. S6). When CMC was reached, we observed a sharp increase in the signal intensity because of the aggregate formation associated with Nile Red 
incorporation in the lipophilic compartment. CMC was determined at different temperatures: we did not find significant differences in $\mathrm{CMC}$ for the studied range, which indicates stability of the assemblies at physiological conditions, including hyperthermia $\left(42^{\circ} \mathrm{C}\right)$.

Previous studies with GAGs side-on modified with thiol reported CMC in the range $30-200 \mu \mathrm{g} / \mathrm{mL}$, depending on the degree of modification and the molecular weight of GAG. $0^{10,28,40,41,43}$ The values that we obtained are at the upper limit of this range or higher, most probably because of the ratio between the GAG and the aliphatic portion: side-on modification is associated with the introduction of several aliphatic moieties per GAG chain, while end-on functionalization results in the introduction of only one aliphatic tail per GAG.

Shelf stability of the micellar nanoparticles is another important property that was monitored by DLS, which is sensitive to small changes in the nanoparticles surface composition ( $\zeta$-potential) and size (PDI). The obtained micellar nanoparticles have strong negative charge associated with long shelf stability, low non-specific binding to plasma proteins, and long circulation half-time. Indeed, our results demonstrated no significant changes either for $\zeta$-potential or for the PDI for one month at $4{ }^{\circ} \mathrm{C}$, i.e. shelf conditions (SI Fig. S7).

Drug encapsulation. Because the micellar nanoparticles have hydrophilic and hydrophobic compartments, we hypothesized that they can be used as carriers of either hydrophilic or lipophilic drugs. We therefore studied the encapsulation efficiency using the fluorescence probes Dextran-FITC (hydrophilic) and Nile Red (lipophilic) as model drugs. Our results demonstrated that Nile Red was encapsulated with $100 \%$ efficiency in the nanoparticles assembled of either HA or CS amphiphile. Dextran-FITC was encapsulated with high efficiency (74.2\%) in HA micellar nanoparticles but with much lower yield (25.2\%) in CS ones. These results are consistent with the characterization of the assemblies described above. The hydrophobic portion of both amphiphiles is the same and the cores of HA or CS nanoparticles are akin. Thus, we expected similar encapsulation efficiency for the lipophilic molecule. The bigger size of the CS nanoparticles is associated with loose packing of the 
CS chains and formation of highly diffusive shell. As a result, the uncharged dextran cannot be encapsulated with high efficiency in this shell. On the other hand, the HA amphiphile assemble in smaller nanoparticles in which the dextran can be efficiently encapsulated due to the establishment of H-bonding with the HA chains.

Redox responsiveness of the micellar nanoparticles. Functionalization of the amphiphile with a thiol group aimed to confer redox responsiveness to the assemblies generated from these amphiphiles. We expected that upon assembly the thiol groups will form disulfide bridges. In the tumor environment, these bridges will break upon the reductive action of the overexpressed GSH triggering the disassembly of the micellar nanoparticles and release of the encapsulated drug. We therefore evaluated the release of Nile Red from the amphiphiles assemblies in the presence of DTT with different concentrations $(0-200 \mathrm{mM})$. We observed different effect for the HA and CS assemblies: increasing concentrations of DTT resulted in a decrease of fluorescence intensity of Nile Red for the HA assemblies, which demonstrates the release of the Nile Red and disassembly of the nanoparticles (SI Fig. S8A, inset) ${ }^{35} \mathrm{~K}_{\text {ap }}$ of each point was determined and the linear fit of the experimental data as a function of DTT concentration (SI Fig. S8A) allowed the determination of the Stern-Volmer $\left(\mathrm{K}_{\mathrm{sv}}\right)$ constant (Table 3). The $\mathrm{K}_{\mathrm{sv}}$ is temperature dependent: temperature increase (from 25 to $37^{\circ} \mathrm{C}$ ) resulted in higher values of $\mathrm{K}_{\mathrm{sv}}$, indicating that the HA assemblies are more sensitive to reductive environment at physiologically relevant temperatures.

Table 3. Stern-Volmer constants for HA micellar nanoparticles obtained by Nile Red fluorescence quenching in presence of increasing concentrations of DTT at different temperatures.

\begin{tabular}{ll}
\hline Temperature $\left[{ }^{\circ} \mathrm{C}\right]$ & $\mathrm{K}_{\mathrm{sv}}\left[\mathrm{M}^{-1}\right]$ \\
\hline 25 & $1.02 \pm 0.40$ \\
37 & $11.80 \pm 0.33$ \\
42 & $14.86 \pm 0.17$ \\
\hline
\end{tabular}


CS assemblies behaved differently: no fluorescence quenching (i.e. Nile Red release) was observed even at the highest concentration of DTT. This result demonstrated that the formation of disulfide bridges between CS amphiphiles is not favored. In fact, this agrees with the structural differences between the GAG assemblies, discussed above: the CS amphiphiles are not packed enough close to allow formation of bridges between the thiol groups.

Redox-sensitivity was also evaluated by DLS. We analyze size distribution of the micellar nanoparticles upon DTT addition (SI Fig. S8B). The results are in good agreement with the fluorescence measurements: clear disassembly is visible for the HA nanoparticles. In the case of CS assemblies, we observed an effect upon initial addition of DTT, but no further variations were detected upon increasing the DTT concentration.

Internalization of the micellar nanoparticles. We used two human breast cancer cell lines that differ by their CD44 expression: MDA-MB-468 overexpress CD44 while SK-BR-3 do not express it (SI Fig. S9). The HA moiety in the synthesized amphiphiles is longer than the required for specific binding of CD44 ( 8 monosaccharides) and thus, we expected that the generated assemblies will be internalized by cells expressing this receptor. ${ }^{4 \& 4}$ The data about the CS-CD44 dependent internalization are controversial; alternative internalization mechanisms are described in addition to CD44 dependent endocytosis. ${ }^{22.50}$ Our results demonstrated that MDA-MB-468 cells internalized both types of particles (Fig. 3A, D) although more intensive red staining was observed for the HA assemblies indicating higher percent of internalization in this case (Fig 3A2 vs 4D2).
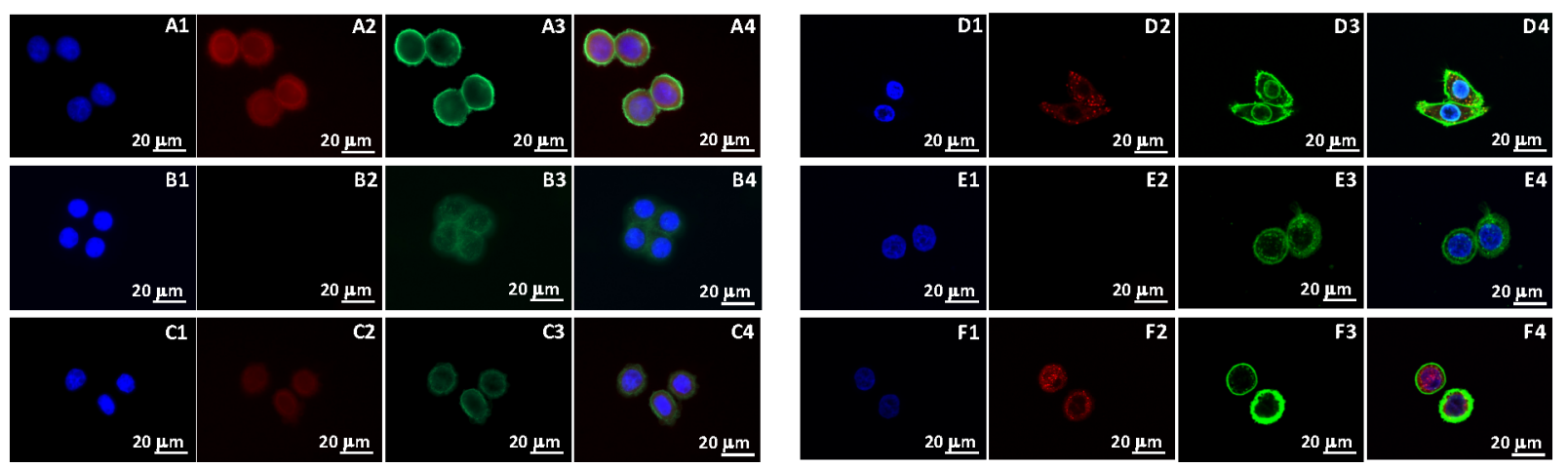
Figure 3. Internalization of HA (A-C) and CS (D-F) micellar nanoparticles loaded with Nile Red by MDA-MB-468 cells without blocking (A, D), after specific CD44 blocking (B, E) and after unspecific IgG1 blocking (C, F). Nuclei staining in blue (1), nanoparticles in red (2), cell membrane in green (3), and merged images (4).

To ensure that this internalization is CD44 mediated, we blocked the CD44 receptors and performed the same assay (Fig. 3B, E). We did not detect any red staining at these conditions indicating no internalization. When using antibodies, however, the internalization could be impeded sterically but not specifically. We therefore performed an additional control experiment in which non-specific blocking was used (IgG1 antibody, Fig. 3C, F). The results demonstrated that although some steric obstruction may take place (lower intensity of Fig. 3C2 vs 3A2), the non-specific blocking did not impede the uptake of the particles by the cells.

Different response was observed when cells that don't express CD44 were brought in contact with the nanoparticles (Fig. 4). SK-BR-3 cells internalized neither HA nor CS assemblies. This result is very important as it shows the selectivity of the developed carriers.
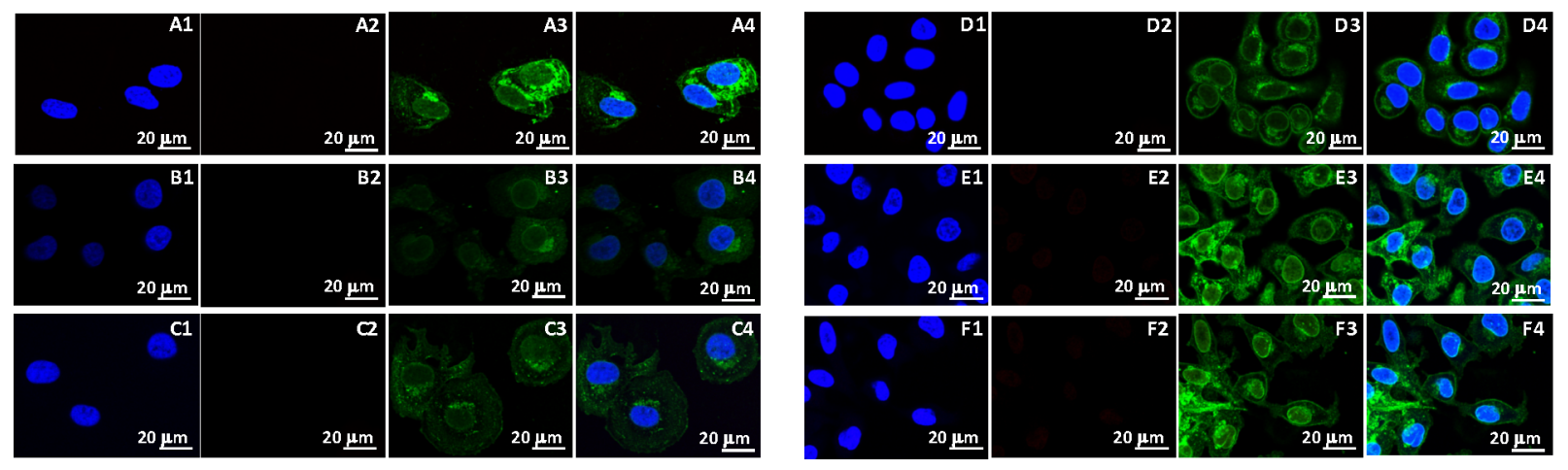

Figure 4. Internalization of HA (A-C) and CS (D-F) micellar nanoparticles loaded with Nile Red by SK-BR-3 cells without blocking (A, D), after specific CD44 blocking (B, E) and after unspecific IgG1 blocking (C, F). Nuclei staining in blue (1), nanoparticles in red (2), cell membrane in green (3), and merged images (4). 


\begin{abstract}
Altogether these data demonstrate that the GAG exposed on the surface of the assemblies is bioactive and interact specifically with the CD44 cell receptors, thus ensuring targeting delivery of the encapsulated cargo.
\end{abstract}

\title{
CONCLUSIONS
}

We demonstrated that amphiphilic GAGs can be obtained by a simple end-on functionalization of different GAGs with aliphatic moiety. The obtained amphiphiles self-assemble in micellar nanoparticles that are stable either at storage or physiological conditions. The size and properties of the assemblies depend on the used GAG: greater encapsulation efficiency and physiological relevant redox responsiveness of the HA nanoparticles make them more suitable as tumor targeting delivery systems. We further showed that the assemblies of HA amphiphiles are recognized by cell expressing CD44 and internalized via specific CD44 pathways. All these features make the HA assemblies very promising as specific nanotheranostic tool targeting CD44 overexpressing cancer cells.

\section{ASSOCIATED CONTENT}

\section{Supporting Information}

Synthesis of 11-(Aminooxy)-undecenethiol and its characterization by NMR; NMR spectra of chondroitin sulfate and its aliphatic derivative; GPC profiles of HA, CS and its aliphatic derivatives; CMC determination by Nile Red fluorescence; HA and CS assemblies' stability by DLS and ELS; redox-sensitivity evaluation by Nile Red fluorescence quenching and by DLS; CD44 expression of MDA-MB-468 and Sk-Br-3 cell lines.

\section{AUTHOR INFORMATION}




\author{
Corresponding author \\ *E-mail: pashkuleva@dep.uminho.pt; Tel: +351.253510906; Fax: +351.253510909 \\ ORCID
}

Iva Pashkuleva: 0000-0001-6818-3374

\title{
Author contributions
}

The manuscript was written through contributions of all authors. All authors have given approval to the final version of the manuscript.

\section{ACKNOWLEDGEMENTS}

The authors thank the Portuguese FCT (Grants no: SFRH/BD/114847/2016, IF/00032/2013 and IF/00373/2014) and EC (ComplexiTE ERC-2012-ADG 20120216-321266, H2020-TWINCHEM2NATURE-692333, H2020-WIDESPREAD-FoReCaST-668983 and EuroNanoMed CytoNanoHeal) for providing financial support to this project.

\footnotetext{
ABBREVIATIONS

HA, hyaluronan; GAGs, glycosaminoglycans; CS, chondroitin sulfate; GSH, glutathione; NMR; nuclear magnetic resonance; GPC, gel permeation chromatography; CMC, critical micelle concentration; DLS, dynamic light scattering; ELS, electrophoretic light scattering; Dh, hydrodynamic diameter; PDI, polydispersity index; AFM, atomic force microscopy; DTT, 1,4-dithiothreitol; KSV, Stern Volmer constant; FITC, fluorescein isothiocyanate; \%EE, encapsulation efficiency; Mw, weightaverage molecular weight; Mn, number-average molecular weight;
} 


\section{REFERENCES}

(1) Hanahan, D.; Weinberg, R. A., The hallmarks of cancer. Cell 2000, 100, (1), 57-70.

(2) Hanahan, D.; Weinberg, R. A., Hallmarks of Cancer: The Next Generation. Cell 2011, 144, (5), 646-674.

(3) Burger, A. M.; Fiebig, H. H., Preclinical screening for new anticancer agents. In Handbook of Anticancer Pharmacokinetics and Pharmacodynamics, Springer: 2014; pp 23-38.

(4) Begley, C. G.; Ellis, L. M., Raise standards for preclinical cancer research. Nature 2012, 483, (7391), 531-533.

(5) Blanco, E.; Shen, H.; Ferrari, M., Principles of nanoparticle design for overcoming biological barriers to drug delivery. Nat Biotechnol 2015, 33, (9), 941-951.

(6) Chen, H. M.; Zhang, W.Z.; Zhu, G.Z.; Xie, J.; Chen, X. Y., Rethinking cancer nanotheranostics. Nat Rev Mater 2017, 2, (5).

(7) Azevedo, H. S.; Pashkuleva, I., Biomimetic supramolecular designs for the controlled release of growth factors in bone regeneration. Adv Drug Deliver Rev 2015, 94, 63-76.

(8) Gu, J.; Chen, X.; Ren, X.; Zhang, X.; Fang, X.; Sha, X., CD44-targeted hyaluronic acid-coated redox-responsive hyperbranched poly (amido amine)/plasmid DNA ternary nanoassemblies for efficient gene delivery. Bioconjugate chemistry 2016, 27, (7), 1723-1736.

(9) Russo, A.; Degraff, W.; Friedman, N.; Mitchell, J. B., Selective Modulation of Glutathione Levels in Human Normal Versus Tumor-Cells and Subsequent Differential Response to Chemotherapy Drugs. Cancer Res 1986, 46, (6), 2845-2848. 
(10) Li, J.; Huo, M. R.; Wang, J.; Zhou, J. P.; Mohammad, J. M.; Zhang, Y. L.; Zhu, Q. N.; Waddad, A. Y.; Zhang, Q., Redox-sensitive micelles self-assembled from amphiphilic hyaluronic aciddeoxycholic acid conjugates for targeted intracellular delivery of paclitaxel. Biomaterials 2012, 33, (7), 2310-2320.

(11) Yu, J.; Chu, X.; Hou, Y. L., Stimuli-responsive cancer therapy based on nanoparticles. Chem Commun 2014, 50, (79), 11614-11630.

(12) Lin, C. W.; Lu, K.-Y.; Wang, S. Y.; Sung, H. W.; Mi, F. L., CD44-specific nanoparticles for redox-triggered reactive oxygen species production and doxorubicin release. Acta Biomater 2016, 35 , 280-292.

(13) Fuster, M. M.; Esko, J. D., The sweet and sour of cancer: Glycans as novel therapeutic targets. Nat Rev Cancer 2005, 5, (7), 526-542.

(14) Rankin, K. S.; Frankel, D., Hyaluronan in cancer - from the naked mole rat to nanoparticle therapy. Soft Matter 2016, 12, (17), 3841-3848.

(15) Chanmee, T.; Ontong, P.; Itano, N., Hyaluronan: A modulator of the tumor microenvironment. Cancer Lett 2016, 375, (1), 20-30.

(16) Wiranowska, M.; Ladd, S.; Moscinski, L. C.; Hill, B.; Haller, E.; Mikecz, K.; Plaas, A., Modulation of hyaluronan production by CD44 positive glioma cells. Int J Cancer 2010, 127, (3), 532542.

(17) da Costa, D. S.; Reis, R. L.; Pashkuleva, I., Sulfation of Glycosaminoglycans and Its Implications in Human Health and Disorders. Annu Rev Biomed Eng 2017, 19, 1-26. 
(18) Bourguignon, L. Y. W.; Shiina, M.; Li, J. J., Hyaluronan-CD44 Interaction Promotes Oncogenic Signaling, microRNA Functions, Chemoresistance, and Radiation Resistance in Cancer Stem Cells Leading to Tumor Progression. Adv Cancer Res 2014, 123, 255-275.

(19) Misra, S.; Hascall, V. C.; Markwald, R. R.; Ghatak, S., Interactions between hyaluronan and its receptors (CD44, RHAMM) regulate the activities of inflammation and cancer. Front Immunol 2015, 6.

(20) Misra, S.; Heldin, P.; Hascall, V. C.; Karamanos, N. K.; Skandalis, S. S.; Markwald, R. R.; Ghatak, S., Hyaluronan-CD44 interactions as potential targets for cancer therapy. Febs $J$ 2011, 278, (9), 1429-1443.

(21) Xin, Y.; Grace, A.; Gallagher, M. M.; Curran, B. T.; Leader, M. B.; Kay, E. W., CD44V6 in gastric carcinoma: A marker of tumor progression. Appl Immunohisto M M 2001, 9, (2), 138-142.

(22) Oommen, O. P.; Duehrkop, C.; Nilsson, B.; Hilborn, J.; Varghese, O. P., Multifunctional Hyaluronic Acid and Chondroitin Sulfate Nanoparticles: Impact of Glycosaminoglycan Presentation on Receptor Mediated Cellular Uptake and Immune Activation. Acs Appl Mater Inter 2016, 8, (32), 20614-20624.

(23) Toole, B. P., Hyaluronan-CD44 Interactions in Cancer: Paradoxes and Possibilities. Clin Cancer Res 2009, 15, (24), 7462-7468.

(24) Song, S. S.; Qi, H.; Xu, J. W.; Guo, P.; Chen, F.; Li, F.; Yang, X. G.; Sheng, N. C.; Wu, Y. L.; Pan, W. S., Hyaluronan-Based Nanocarriers with CD44-Overexpressed Cancer Cell Targeting. Pharm Res-Dordr 2014, 31, (11), 2988-3005.

(25) Kang, B.; Opatz, T.; Landfester, K.; Wurm, F. R., Carbohydrate nanocarriers in biomedical applications: functionalization and construction. Chem Soc Rev 2015, 44, (22), 8301-8325. 
(26) Mattheolabakis, G.; Milane, L.; Singh, A.; Amiji, M. M., Hyaluronic acid targeting of CD44 for cancer therapy: from receptor biology to nanomedicine. J Drug Target 2015, 23, (7-8), 605-618.

(27) Vafaei, S. Y.; Esmaeili, M.; Amini, M.; Atyabi, F.; Ostad, S. N.; Dinarvand, R., Self assembled hyaluronic acid nanoparticles as a potential carrier for targeting the inflamed intestinal mucosa. Carbohyd Polym 2016, 144, 371-381.

(28) Lin, T. S.; Yuan, A.; Zhao, X. Z.; Lian, H. B.; Zhuang, J. L.; Chen, W.; Zhang, Q.; Liu, G. X.; Zhang, S. W.; Chen, W.; Cao, W. M.; Zhang, C. W.; Wu, J. H.; Hu, Y. Q.; Guo, H. Q., Self-assembled tumor-targeting hyaluronic acid nanoparticles for photothermal ablation in orthotopic bladder cancer. Acta Biomater 2017, 53, 427-438.

(29) Altgarde, N.; Nileback, E.; de Battice, L.; Pashkuleva, I.; Reis, R. L.; Becher, J.; Moller, S.; Schnabelrauch, M.; Svedhem, S., Probing the biofunctionality of biotinylated hyaluronan and chondroitin sulfate by hyaluronidase degradation and aggrecan interaction. Acta Biomater 2013, 9, (9), 8158-8166.

(30) Silva, C.; Carretero, A.; da Costa, D. S.; Reis, R. L.; Novoa-Carballal, R.; Pashkuleva, I., Design of protein delivery systems by mimicking extracellular mechanisms for protection of growth factors. Acta Biomater 2017, 63, 283-293.

(31) Novoa-Carballal, R.; Silva, C.; Moller, S.; Schnabelrauch, M.; Reis, R. L.; Pashkuleva, I., Tunable nano-carriers from clicked glycosaminoglycan block copolymers. J Mater Chem B 2014, 2, (26), 4177-4184.

(32) Upadhyay, K. K.; Bhatt, A. N.; Mishra, A. K.; Dwarakanath, B. S.; Jain, S.; Schatz, C.; Le Meins, J. F.; Farooque, A.; Chandraiah, G.; Jain, A. K.; Misra, A.; Lecommandoux, S., The intracellular drug delivery and anti tumor activity of doxorubicin loaded poly(gamma-benzyl Lglutamate)-b-hyaluronan polymersomes. Biomaterials 2010, 31, (10), 2882-2892. 
(33) Upadhyay, K. K.; Le Meins, J. F.; Misra, A.; Voisin, P.; Bouchaud, V.; Ibarboure, E.; Schatz, C.; Lecommandoux, S., Biomimetic Doxorubicin Loaded Polymersomes from Hyaluronan-blockPoly(gamma-benzyl glutamate) Copolymers. Biomacromolecules 2009, 10, (10), 2802-2808.

(34) Silva, C.; Novoa-Carballal, R.; Reis, R. L.; Pashkuleva, I., Following the enzymatic digestion of chondroitin sulfate by a simple GPC analysis. Anal Chim Acta 2015, 885, 207-213.

(35) Kurniasih, I. N.; Liang, H.; Mohr, P. C.; Khot, G.; Rabe, J. P.; Mohr, A., Nile Red Dye in Aqueous Surfactant and Micellar Solution. Langmuir 2015, 31, (9), 2639-2648.

(36) Shlyakhtenko, L. S.; Gall, A. A.; Filonov, A.; Cerovac, Z.; Lushnikov, A.; Lyubchenko, Y. L., Silatrane-based surface chemistry for immobilization of DNA, protein-DNA complexes and other biological materials. Ultramicroscopy 2003, 97, (1-4), 279-287.

(37) Peluso, S.; Imperiali, B., Asparagine surrogates for the assembly of N-linked glycopeptide mimetics by chemoselective ligation. Tetrahedron Lett 2001, 42, (11), 2085-2087.

(38) Novoa-Carballal, R.; Muller, A. H. E., Synthesis of polysaccharide-b-PEG block copolymers by oxime click. Chem Commun 2012, 48, (31), 3781-3783.

(39) Thakar, D.; Migliorini, E.; Coche-Guerente, L.; Sadir, R.; Lortat-Jacob, H.; Boturyn, D.; Renaudet, O.; Labbe, P.; Richter, R. P., A quartz crystal microbalance method to study the terminal functionalization of glycosaminoglycans. Chem Commun 2014, 50, (96), 15148-15151.

(40) Pitarresi, G.; Palumbo, F. S.; Albanese, A.; Fiorica, C.; Picone, P.; Giammona, G., Selfassembled amphiphilic hyaluronic acid graft copolymers for targeted release of antitumoral drug. $J$ Drug Target 2010, 18, (4), 264-276. 
(41) Liu, H. X.; Wu, S. Q.; Yu, J. M.; Fan, D.; Ren, J.; Zhang, L.; Zhao, J. G., Reduction-sensitive micelles self-assembled from amphiphilic chondroitin sulfate A-deoxycholic acid conjugate for triggered release of doxorubicin. Mat Sci Eng C-Mater 2017, 75, 55-63.

(42) Zhang, H.; Xu, J. K.; Xing, L.; Ji, J. B.; Yu, A. H.; Zhai, G. X., Self-assembled micelles based on Chondroitin sulfate/poly (D,L-lactideco-glycolide) block copolymers for doxorubicin delivery. $J$ Colloid Interf Sci 2017, 492, 101-111.

(43) Liu, Y. H.; Sun, J.; Cao, W.; Yang, J. H.; Lian, H.; Li, X.; Sun, Y. H.; Wang, Y. J.; Wang, S. L.; He, Z. G., Dual targeting folate-conjugated hyaluronic acid polymeric micelles for paclitaxel delivery. Int J Pharmaceut 2011, 421, (1), 160-169.

(44) Wu, J. L.; Liu, C. G.; Wang, X. L.; Huang, Z. H., Preparation and characterization of nanoparticles based on histidine-hyaluronic acid conjugates as doxorubicin carriers. J Mater Sci-Mater M 2012, 23, (8), 1921-1929.

(45) Teixeira, R.; Reis, R. L.; Pashkuleva, I., Influence of the sulfation degree of glycosaminoglycans on their multilayer assembly with poly-L-lysine. Colloid Surface B 2016, 145, 567-575.

(46) Park, H. K.; Lee, S. J.; Oh, J. S.; Lee, S. G.; Jeong, Y. I. L.; Lee, H. C., Smart Nanoparticles Based on Hyaluronic Acid for Redox-Responsive and CD44 Receptor-Mediated Targeting of Tumor. Nanoscale Research Letters 2015, 10, (1), 288.

(47) Topel, O.; Cakir, B. A.; Budama, L.; Hoda, N., Determination of critical micelle concentration of polybutadiene-block-poly(ethyleneoxide) diblock copolymer by fluorescence spectroscopy and dynamic light scattering. J Mol Liq 2013, 177, 40-43.

(48) Louderbough, J. M. V.; Schroeder, J. A., Understanding the Dual Nature of CD44 in Breast Cancer Progression. Mol Cancer Res 2011, 9, (12), 1573-1586. 
(49) Sheridan, C.; Kishimoto, H.; Fuchs, R. K.; Mehrotra, S.; Bhat-Nakshatri, P.; Turner, C. H.; Goulet, R.; Badve, S.; Nakshatri, H., CD44(+)/CD24(-) breast cancer cells exhibit enhanced invasive properties: an early step necessary for metastasis. Breast Cancer Res 2006, 8, (5).

(50) Pathak, A.; Kumar, P.; Chuttani, K.; Jain, S.; Mishra, A. K.; Vyas, S. P.; Gupta, K. C., Gene Expression, Biodistribution, and Pharmacoscintigraphic Evaluation of Chondroitin Sulfate-PEI Nanoconstructs Mediated Tumor Gene Therapy. Acs Nano 2009, 3, (6), 1493-1505. 
TOC
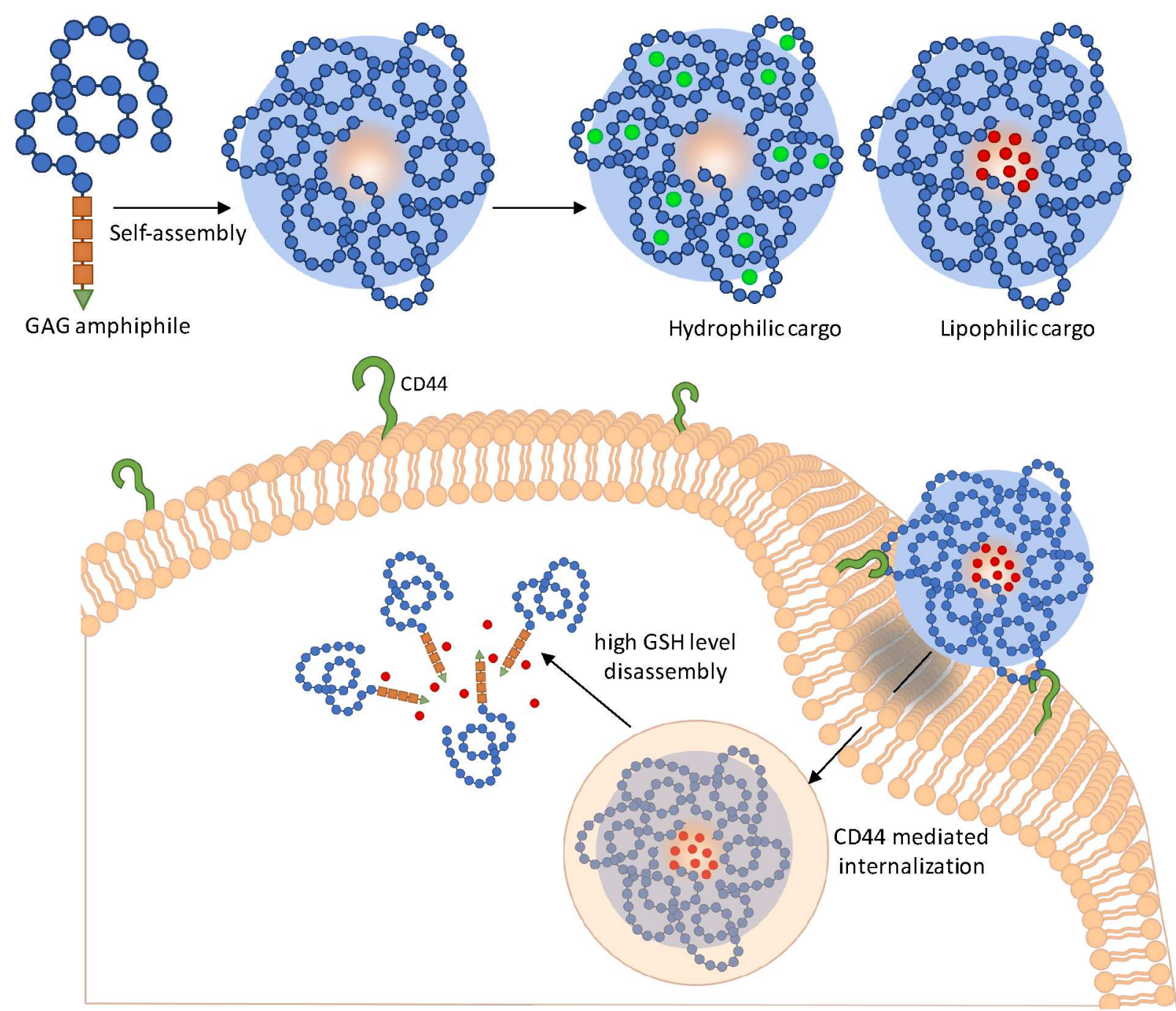